\title{
Comparative effectiveness of treatment options for plantar heel pain: a systematic review with network meta-analysis
}

\author{
Opeyemi 0 Babatunde, ${ }^{1}$ Amardeep Legha, ${ }^{1,2}$ Chris Littlewood, ${ }^{1}$ Linda S Chesterton, ${ }^{1}$ \\ Martin J Thomas, ${ }^{1,3}$ Hylton B Menz, ${ }^{1,4}$ Danielle van der Windt, ${ }^{1,2}$ Edward Roddy ${ }^{1,3}$
}

\begin{abstract}
- Additional material is published online only. To view, please visit the journal online (http://dx.doi.org/10.1136/ bjsports-2017-098998).

'Research Institute for Primary Care \& Health Sciences, Arthritis Research UK Primary Care Centre, Keele, Staffordshire, UK ${ }^{2}$ Centre for Prognosis Research, Research Institute for Primary Care and Health Sciences, Keele University, Staffordshire, UK ${ }^{3}$ Haywood Academic Rheumatology Centre, Staffordshire and Stoke-on-Trent Partnership NHS Trust, Haywood Hospital, Burslem, Staffordshire, UK

${ }^{4} S$ chool of Allied Health, College of Science, Health and Engineering, La Trobe University, Bundoora Victoria, Australia
\end{abstract}

Correspondence to Dr Opeyemi 0 Babatunde, Arthritis Research UK Primary Care Centre, Research Institute for Primary Care \& Health Sciences, Keele University, Staffordshire, ST5 5BG, UK; o.babatunde@keele.ac.uk

Accepted 1 June 2018 Published Online First 28 June 2018

Check for updates

(C) Author(s) (or their employer(s)) 2019. No commercial re-use. See rights and permissions. Published by BMJ.

To cite: Babatunde 00 , Legha A, Littlewood C, et al. Br I Sports Med 2019:53:182-194.

\section{ABSTRACT}

Objective To evaluate the comparative effectiveness of current treatment options for plantar heel pain (PHP).

Design Systematic review and network meta-analysis (NMA).

Data sources Medline, EMBASE, CINAHL, AMED, PEDro, Cochrane Database, Web of Science and WHO Clinical Trials Platform were searched from their inception until January 2018.

Study selection Randomised controlled trials (RCTs) of adults with PHP investigating common treatments (ie, corticosteroid injection, non-steroidal antiinflammatory drugs, therapeutic exercise, orthoses and/ or extracorporeal shockwave therapy (ESWT)) compared with each other or a no treatment, placebo/sham control. Data extraction and analysis Data were extracted and checked for accuracy and completeness by pairs of reviewers. Primary outcomes were pain and function. Comparative treatment effects were analysed by random effects NMA in the short term, medium term and long term. Relative ranking of treatments was assessed by surface under the cumulative ranking probabilities (0-100 scale).

Results Thirty-one RCTs (total $n=2450$ patients) were included. There was no evidence of inconsistency detected between direct and indirect treatment comparisons in the networks, but sparse data led to frequently wide Cls. Available evidence does not suggest that any of the commonly used treatments for the management of PHP are better than any other, although corticosteroid injections, alone or in combination with exercise, and ESWT were ranked most likely to be effective for the management of short-term, mediumterm and long-term pain or function; placebo/sham/ control appeared least likely to be effective; and exercise appeared to only be beneficial for long-term pain or function.

Conclusions Current evidence is equivocal regarding which treatment is the most effective for the management of PHP. Given limited understanding of long-term effects, there is need for large, methodologically robust multicentre RCTs investigating and directly comparing commonly used treatments for the management of PHP.

PROSPERO registration number CRD42016046963.

\section{INTRODUCTION}

Plantar heel pain (PHP) is the most prevalent soft tissue foot complaint, affecting $10 \%$ of adults during their lifetime ${ }^{1}$ and accounting for $25 \%$ of all foot disorders in athletes. ${ }^{2}$ Characterised by insidious onset, localised pain in the plantar heel region which may extend to the medial arch of the foot, the cause of PHP is unclear but is likely multifactorial. Risk factors include obesity, pronated foot type, reduced ankle or first metatarsophalangeal joint range of motion and prolonged weightbearing. ${ }^{3-5}$ PHP reduces mobility, impairs foot and physical function and the capacity for work, all of which have a negative impact on health-related quality of life. ${ }^{167}$

In terms of primary care management, current guidance suggests a period of watchful waiting with self-management advice followed by conservative interventions if there is no improvement, including; therapist-led exercises, foot orthoses, corticosteroid injections and extracorporeal shockwave therapy (ESWT) ${ }^{8-10}$ Although PHP is commonly thought to be a self-limiting condition, resolution of symptoms in some patients may take up to 18 months. ${ }^{11}$ Research to date suggests treatments do offer potential benefits in terms of reduced pain and improved function, ${ }^{1}$ but clinical decision-making is hampered due to a lack of robust evidence to inform the choice of treatment.

A Cochrane systematic review ${ }^{12}$ considered a range of interventions (including exercises, foot orthoses, corticosteroid injections, ESWT, laser therapy and therapeutic ultrasound) for PHP, but was not able to pool the available data, found inconclusive evidence for the effectiveness of treatments and overall, found limited evidence to inform clinical practice. Since the publication of this review, a number of additional randomised controlled trials (RCTs) have been conducted, of which the evidence has yet to be synthesised. A recent review ${ }^{13}$ of conservative treatments for $\mathrm{PHP}$ included many interventions (eg, laser therapy, orthoses, pulsed radiofrequency, dry-needling), which are not commonly used for managing PHP, and analyses were limited by lack of power (two to three studies, mostly small sample sizes) except for the ESWT versus placebo comparison. Also, other previous systematic reviews ${ }^{10} 12$ 14-16 have focused mostly on pairwise comparisons of two or three treatment options.

Day-to-day clinical decision making, however, often involves consideration of the 'most effective' among available treatment options for PHP. Network meta-analysis (NMA) as a novel synthesis of evidence allows for simultaneous inferences regarding clinical effectiveness of all available treatment options, by drawing together evidence from 


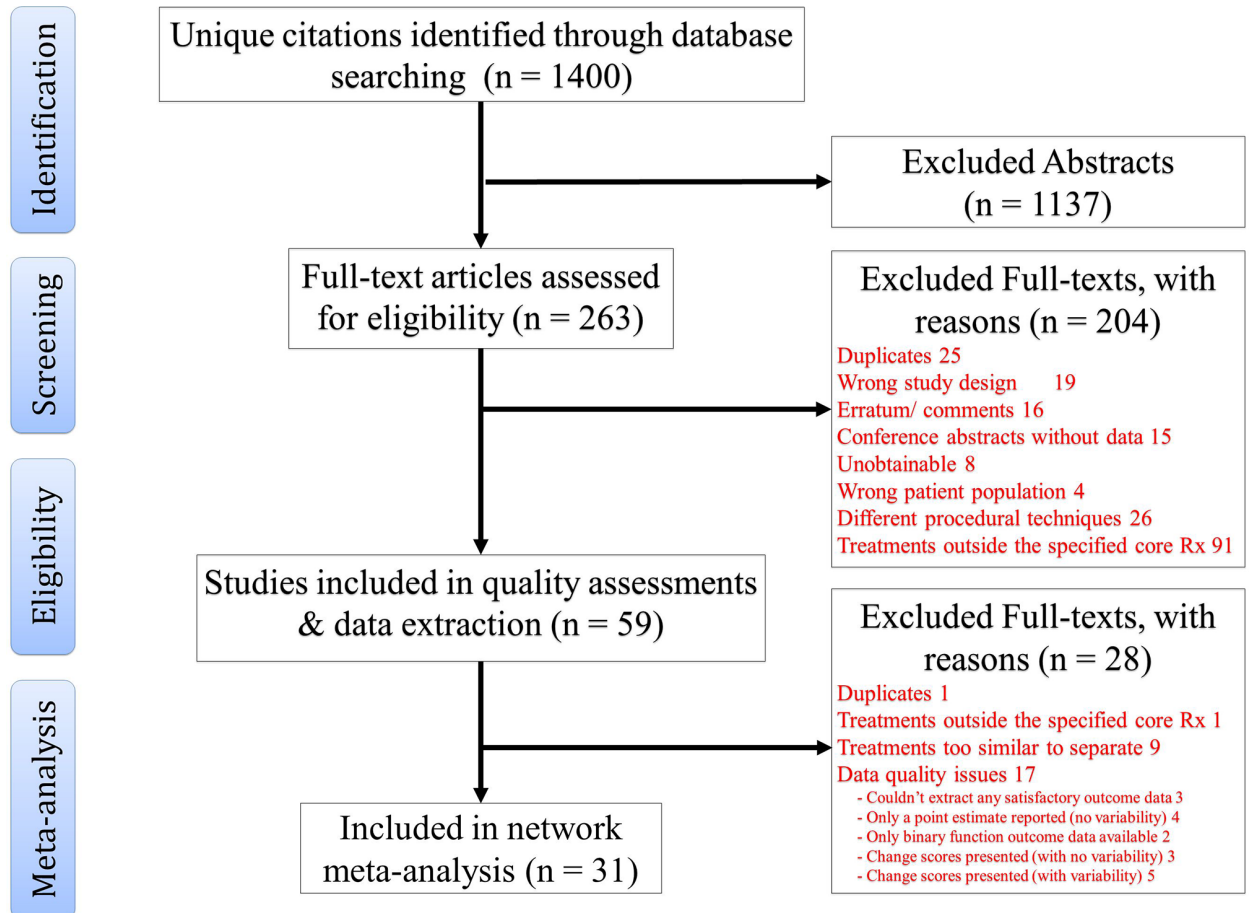

Figure 1 Study flow chart.

direct and indirect comparisons of multiple treatments. ${ }^{17} \mathrm{Com}-$ pared with traditional pairwise comparisons, NMA has the potential to increase the precision of the estimates of effects. Also, NMA enables a ranking of the different treatments relative to each other and aids clinical/shared decision making for clinicians and patients who may desire to know the 'best treatment' on average. ${ }^{17}$

There is a need therefore, to undertake a comprehensive, up-to-date systematic review of the comparative effectiveness of treatment options for PHP. Using an NMA, this study aimed to evaluate and compare the most common conservative treatment options for the management of PHP.

The specific objectives of this study were to:

i. determine the comparative effectiveness of treatments for relieving pain and improving function in patients with PHP;

ii. identify gaps in the available evidence, as well as identify promising treatments that require investigation in future RCTs.

\section{METHODS}

\section{Protocol/protocol registration}

This review was conducted and reported in accordance with the Preferred Reporting Items for Systematic Reviews and Meta-Analyses extension statement for systematic reviews incorporating network meta-analyses for healthcare. ${ }^{18}$ An a priori protocol was established for this review and registered with the international prospective register of systematic reviews, PROSPERO number CRD42016046963 (http://www.crd.york.ac.uk/ PROSPERO/display_record.php?ID=CRD42016046963).

\section{Patient involvement}

Patient involvement was central to the development of the research question. Within an advisory workshop which included participants who currently have or have experienced PHP $(n=6)$ and clinicians $(n=12$; physiotherapists and podiatrists) involved in the management of foot pain, patients discussed their experiences of PHP and their concerns about the need to determine effective treatment options for relieving symptoms and improving function (ie, pain-free walking).

\section{Study eligibility}

We evaluated each identified RCT against the following predetermined selection criteria:

i. Study population: adults, aged 18 years and older with PHP (including plantar fasciitis, plantar fasciopathy, plantar fasciosis) as diagnosed by clinical examination and/or diagnostic imaging.

ii. Interventions: the review focused on four therapeutic interventions (ie, exercise therapy, corticosteroid injections, non-steroidal anti-inflammatory drugs (NSAIDs) and orthoses) that are commonly used in the management of PHP in the $\mathrm{UK}^{19}$ and an additional treatment (ie, ESWT), which is commonly reported in the literature.

iii. Due to an envisaged lack of suitable data on dosage and procedural variations of treatment options, this systematic review and NMA focused primarily on comparisons of the specified core therapeutic interventions (exercise therapy, corticosteroid injections, orthoses, NSAIDs and ESWT).

iv. Comparator: direct comparisons between any of the five core therapeutic interventions (ie, exercise therapy, corticosteroid injections, NSAIDs, orthoses and ESWT) or comparisons with usual care/placebo/sham for PHP in any healthcare setting (community, primary healthcare or secondary healthcare), and without restrictions regarding duration, frequency or intensity of treatment. Studies only comparing different procedural techniques of the same intervention (eg, focal vs radial shockwave) were excluded.

v. Outcome measure: the primary outcomes for this review were pain and functional disability. In order to be eligible for inclusion, assessment of pain and/or functional disability was required, studies with $<24$ hours follow-up were excluded. Pain measures were placed in a hierarchy as follows: 


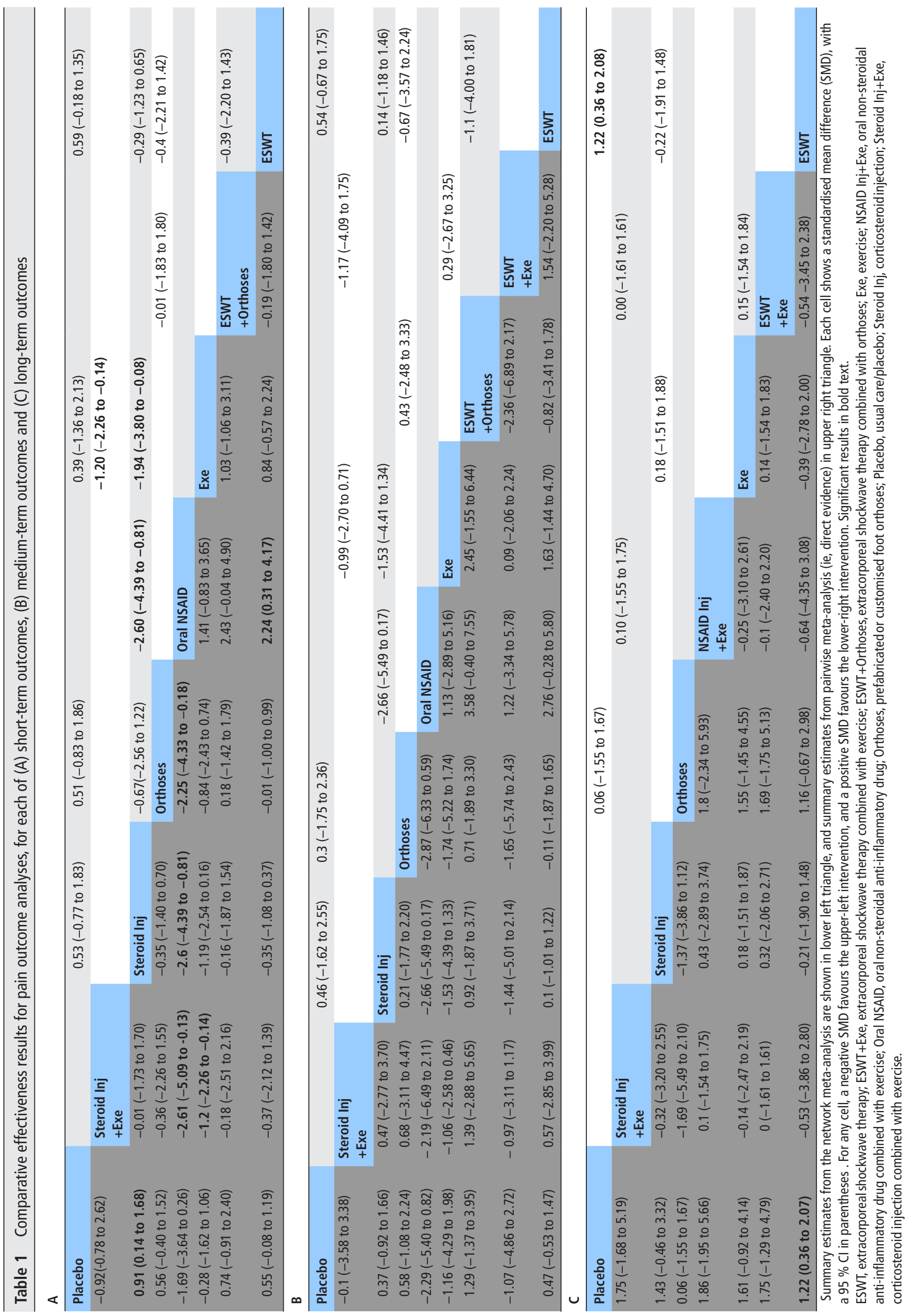


Table 2 Network meta-analysis treatment ranking results for pain outcome analyses, for each of short-term outcomes, medium-term outcomes and long-term outcomes

\begin{tabular}{|c|c|c|c|c|c|c|}
\hline \multirow[b]{2}{*}{ Treatment } & \multicolumn{2}{|c|}{ Short-term pain } & \multicolumn{2}{|c|}{ Medium-term pain } & \multicolumn{2}{|c|}{ Long-term pain } \\
\hline & SUCRA & Mean rank & SUCRA & Mean rank & SUCRA & Mean rank \\
\hline ESWT & 60.7 & 3.8 & 67.2 & 3.6 & 54.5 & 4.2 \\
\hline ESWT+Exe & & & 29.4 & 6.6 & 64.2 & 3.5 \\
\hline ESWT+Orthoses & 66.5 & 3.3 & 80.3 & 2.6 & & \\
\hline Exe & 24.6 & 6.3 & 26.1 & 6.9 & 61.4 & 3.7 \\
\hline NSAID Inj+Exe & & & & & 63.3 & 3.6 \\
\hline Oral NSAID & 3.7 & 7.7 & 13.3 & 7.9 & & \\
\hline Orthoses & 60.5 & 3.8 & 66.6 & 3.7 & 20 & 6.6 \\
\hline Placebo & 30.1 & 5.9 & 48.7 & 5.1 & 15.6 & 6.9 \\
\hline Steroid Inj & 79.5 & 2.4 & 63.7 & 3.9 & 58.4 & 3.9 \\
\hline Steroid Inj+Exe & 74.4 & 2.8 & 54.7 & 4.6 & 62.7 & 3.6 \\
\hline
\end{tabular}

Surface under cumulative ranking curve (SUCRA) values $(0-100)$ and mean ranks are presented, based on a simulation with 1000 replications. Higher SUCRA s and lower mean ranks indicate better performing treatments.

ESWT, extracorporeal shockwave therapy; ESWT+Exe, extracorporeal shockwave therapy combined with exercise; ESWT+Orthoses, extracorporeal shockwave therapy combined with orthoses; Exe, exercise; NSAID Inj+Exe, oral non-steroidal anti-inflammatory drug combined with exercise; Oral NSAID, oral non-steroidal anti-inflammatory drug; Orthoses, prefabricated or customised foot orthoses; Placebo, usual care/placebo; Steroid Inj, corticosteroid injection; Steroid Inj+Exe, corticosteroid injection combined with exercise.

first step pain, pain in the morning, pain on activity (eg, walking), overall pain (or other measures of pain). This hierarchy was used to analyse the most clinically relevant data when multiple pain outcomes were reported in an RCT.

\section{Information sources and search strategy}

A comprehensive search strategy was developed in collaboration with an information specialist, with input from clinicians and academics in the review team. Eight electronic databases (Medline, EMBASE, CINAHL, AMED, PEDro, Cochrane Database of Systematic Reviews Cochrane Controlled Clinical Trials (CENTRAL), Web of Science and WHO International Clinical Trials Registry Platform) were searched from their inception until January 2018 (see online supplementary appendix 1 for full search strategies). No language restrictions were applied. The bibliographies of relevant review articles and selected articles were examined for additional potentially relevant trials.

\section{Study selection}

In pairs, reviewers (OB, AL, CL, LSC, MJT, DvdW, ER) independently evaluated the eligibility of identified trials. At each stage of titles, abstracts and full-text selection, discrepancies were resolved through discussion between pairs of reviewers or via consensus in review team meetings.

\section{Risk of bias assessment}

The Cochrane Collaboration's Risk of Bias tool ${ }^{20}$ was used to assess the quality of included trials. Trials were graded (unclear, high or low risk of bias) based on: (i) sequence generation, (ii) allocation concealment, (iii) blinding of personnel, (iv) blinding of outcome assessor, (v) incomplete outcome data, (vi) selective outcome reporting and (vii) other bias. For each study, risk of bias items was judged as unclear when there was either insufficient information to judge as (low/high risk) or there was no related information regarding the risk of bias item in the report (further details on risk of bias assessment are presented in online supplementary appendix 1).

\section{Data extraction}

Using a customised, pretested and piloted data extraction form, risk of bias and data extraction for each included trial were performed by pairs of reviewers. Differences in quality appraisal and extracted data were resolved through discussion between pairs of reviewers and where appropriate, the opinion of other members of the review team. For each included trial, details were extracted on: design, sample size, population characteristics (eg, age, diagnosis, duration of heel pain, interventions (professional delivering intervention, dose, duration and number of sessions) and outcome assessment (type of outcome measure, length of follow-up and outcome measurements)). Studies that provided a point estimate of the outcome together with a measure of variability (eg, a mean and SD), were taken forward for analysis. Where only sample size, median, range and/or IQR was given, methodology from Wan $e t a l^{21}$ was used to calculate the sample mean and SD. In instances of missing or incomplete data (eg, lack of measures of variability for follow-up data), additional information was requested and obtained (where possible) through contacting primary study authors.

\section{Data synthesis and analysis}

All analyses were performed using STATA V.15.1 (StataCorp, Texas, USA), under a frequentist approach, with restricted maximum likelihood used to estimate parameters. Prior to analyses, extracted data were further checked independently for completion and accuracy by the study statistician while profiling a database for the analyses. Furthermore, in order to define the treatment nodes for the network, two reviewers (HBM and ER), a podiatrist and rheumatologist, independently reviewed and classified the therapeutic interventions following a consensus process. As the objective of this systematic review was to compare different treatment options, and not to investigate the influence of dosage or intensity of interventions, the specified core therapeutic interventions (exercise therapy, corticosteroid injections, orthoses, NSAIDs, and ESWT), and usual care/placebo/sham, were allocated to six distinct nodes. Furthermore, studies involving combination(s) of any of the specified core treatments were used in our analyses in addition to the six nodes as treatment nodes with combination treatments. For example, where trial arms have involved a combination of exercise therapy and a corticosteroid injection as an intervention, corticosteroid injection + exercise was classed as a distinct treatment node. Also, where RCTs included more than one arm with 

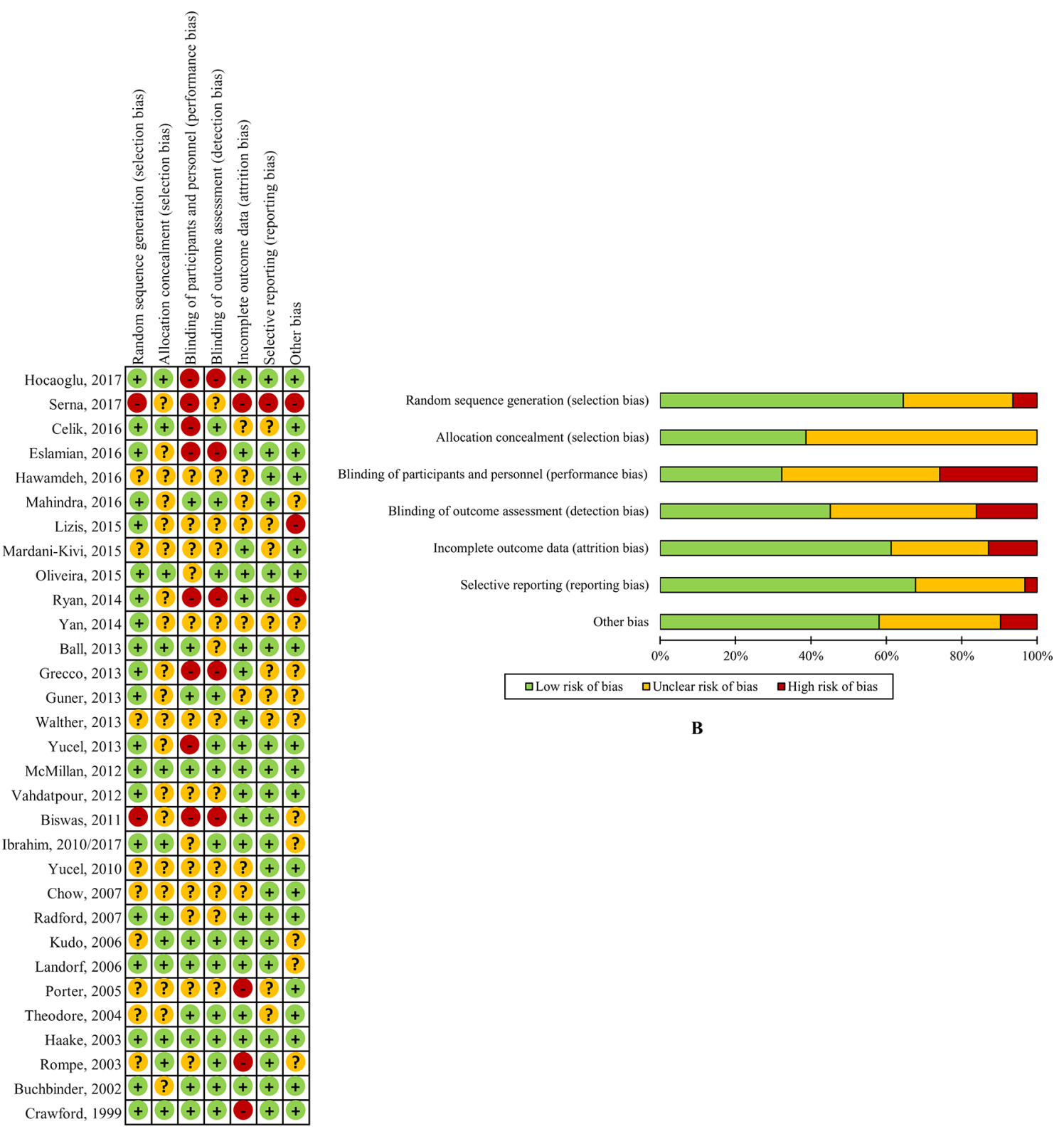

B

Figure 2 (A) Risk of bias for all individual studies $(n=31)$ included in the analysis. (B) Summary of risk of bias across all $(n=31)$ studies included in the analysis. + (green circle), low risk of bias; ? (amber circle), unclear risk of bias; - (red circle), high risk of bias.

the same type of treatment, the data were pooled together (eg, for a three-armed trial ${ }^{22}$ involving a prefabricated orthoses arm, and two custom orthoses arms (differentiated by a rigid and soft material), an average of the mean outcomes and SD was taken from the custom orthoses arms and a sum taken from the arm sample sizes, in order to create a single pooled orthoses arm).

In order to obtain direct treatment effect estimates (with a 95\% CI) for each included comparison, pairwise meta-analyses were performed. Direct and indirect estimates of effects were then analysed together in an NMA.

\section{Network coherence (consistency and heterogeneity)}

The important assumption underlying an NMA is that of network consistency, that is, true treatment effects are on average the same, regardless of whether they are estimated from direct or indirect evidence. This was assessed in three ways: (i) using a global Wald test (with high $\mathrm{p}$ values favouring consistency) ${ }^{23}$; (ii) using a node-splitting technique, which judges the consistency of direct and indirect estimates separately for each treatment comparison (with high $\mathrm{p}$ values favouring consistency) ${ }^{24}$ and (iii) graphically (as a crude test), by inspection of forest plots comparing direct and pooled NMA results. Furthermore, the choice of a random or fixed effects model for each analysis was based on the magnitude of $\tau^{2}$ (ie, the common betweenstudy variance across all treatment comparisons). A structured between-studies variance-covariance matrix was used, which assumes that all treatment comparisons have a common heterogeneity variance.

Primary outcomes of pain and function

Primary outcomes of pain and function were classified as: (i) short term ( 1 to $\leq 6$ weeks post-treatment), (ii) medium term ( 6 to $\leq 12$ weeks post-treatment) or (iii) long term ( $>12$ weeks post-treatment). For short-term and medium-term outcomes, the 
Table 3 Comparative effectiveness results for function outcome analyses, for each of (A) short-term outcomes, (B) medium-term outcomes and (C) long-term outcomes

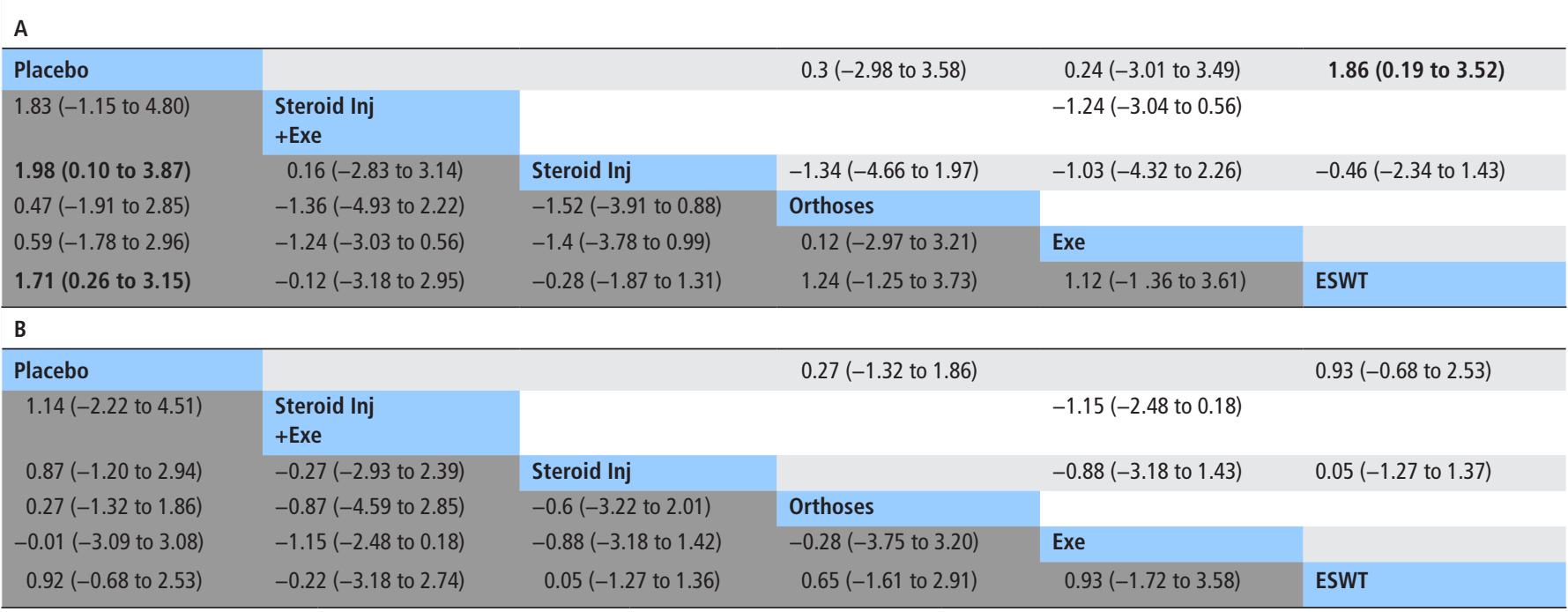

C

\begin{tabular}{|c|c|c|c|c|}
\hline Placebo & & $0.11(-0.26$ to 0.47$)$ & & $0.95(0.50$ to 1.40$)$ \\
\hline $0.93(0.23$ to 1.63$)$ & Steroid Inj & & $0.16(-0.47$ to 0.79$)$ & $0.02(-0.51$ to 0.56$)$ \\
\hline $0.11(-0.26$ to 0.47$)$ & $-0.82(-1.61$ to -0.03$)$ & Orthoses & & \\
\hline 1.09 (0.15 to 2.03$)$ & $0.16(-0.47$ to 0.79$)$ & $0.98(-0.03$ to 1.99$)$ & Exe & \\
\hline $0.95(0.50$ to 1.40$)$ & $0.03(-0.51$ to 0.56$)$ & 0.84 (0.26 to 1.43$)$ & $-0.14(-0.96$ to 0.69$)$ & ESWT \\
\hline
\end{tabular}

Summary estimates from the network meta-analysis are shown in lower left triangle, and summary estimates from pairwise meta-analysis (ie, direct evidence) in upper right triangle. Each cell shows a standardised mean difference (SMD), with a $95 \% \mathrm{Cl}$ in parentheses. For any cell, a negative SMD favours the upper-left intervention, and a positive SMD favours the lower-right intervention. Significant results in bold text.

ESWT, extracorporeal shockwave therapy; Exe, exercise; Orthoses, prefabricated or customised foot orthoses; Placebo, usual care/placebo; Steroid Inj, corticosteroid injection; Steroid Inj+Exe, corticosteroid injection combined with exercise.

latest outcome data within each time category was used for analysis. For example, if a study reported 3-week and 6-week pain outcomes, only the 6-week data were used. However, because the long-term category has no upper bound, a different approach was taken to reduce potential heterogeneity in results; we evaluated the spread of long-term outcomes and selected the most prevalent time point, and only retained data matching this time point for analysis. A total of six NMAs were possible (pain or function outcomes analysed separately for each time category), and a network plot was used to graphically present the direct evidence base and assess connectedness of each network.

\section{Assessing comparative effectiveness of treatments}

The principal summary measure used for pain and function outcomes was the standardised mean difference (SMD). SMDs are advantageous in homogenising outcomes from different scales and instruments onto a common scale. The direction of outcome scales in the raw data were reversed where appropriate (by multiplying values by -1 ), to ensure all outcomes were interpreted with lower values indicative of improvements in pain or functional disability. Estimates of effects (SMDs) were interpreted according to Cohen's rule of thumb, with values of $0.2,0.5$ and 0.8 indicative of small, moderate and large effects, respectively. ${ }^{25}$ Direct pairwise (where available) and pooled NMA estimates, along with 95\% CIs, are reported for all treatment comparisons. SMDs with $95 \%$ CIs that did not include the null value (of $\mathrm{SMD}=0$, ie, no difference in comparative treatment effect), were classed as statistically significant.

\section{Ranking of treatments}

To further assess the comparative effectiveness of treatments, the ranking probability distributions of each treatment were generated from a simulation of 1000 replications. We used mean rank, surface under cumulative ranking curve (SUCRA) values and cumulative ranking plots. These statistics rank treatments according to their ability to generate the largest treatment effects in each simulation, and are averaged over the 1000 replications.

\section{Sensitivity analysis}

To assess the robustness of the findings for pain and functional outcomes, sensitivity analysis based on risk of bias was planned but not performed. This was due to most studies showing similar (unclear) risks of bias. Sensitivity analysis by the removal of studies with unclear risk led to insufficient data to support the network.

\section{RESULTS}

\section{Characteristics of included studies}

The literature search yielded 1400 unique citations, of which 263 full-text articles were selected for full review. The study flow chart is presented in figure 1. Of the 263 full-text articles, 59 met the inclusion criteria and were subjected to quality assessment and data extraction. A further 28 articles could not be included in the NMA due to: being duplicate reports of the same RCT $(n=1)$; examining dose regimen/technique comparisons of the same intervention $(n=9)$; examining a similar but different treatment to commonly used interventions for PHP, that is, intracorporeal pneumatic shock therapy $(n=1)$ and 
A

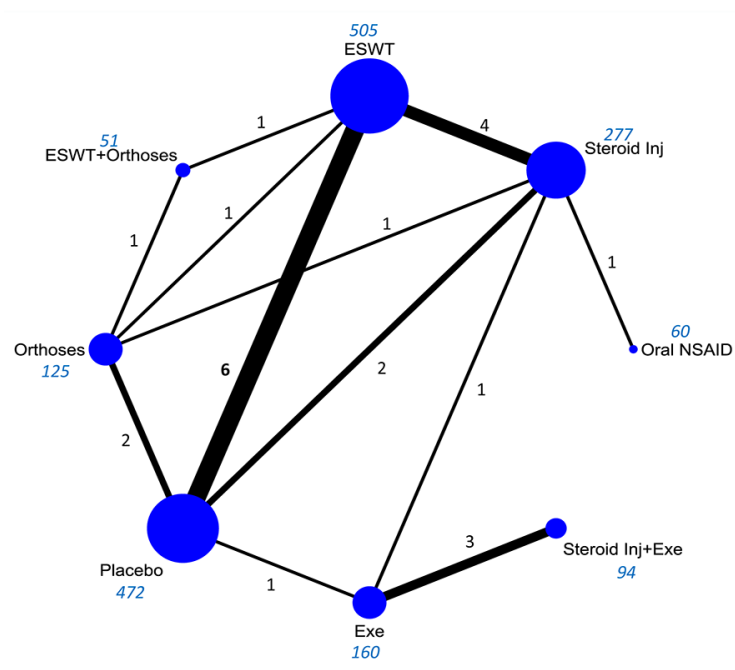

C

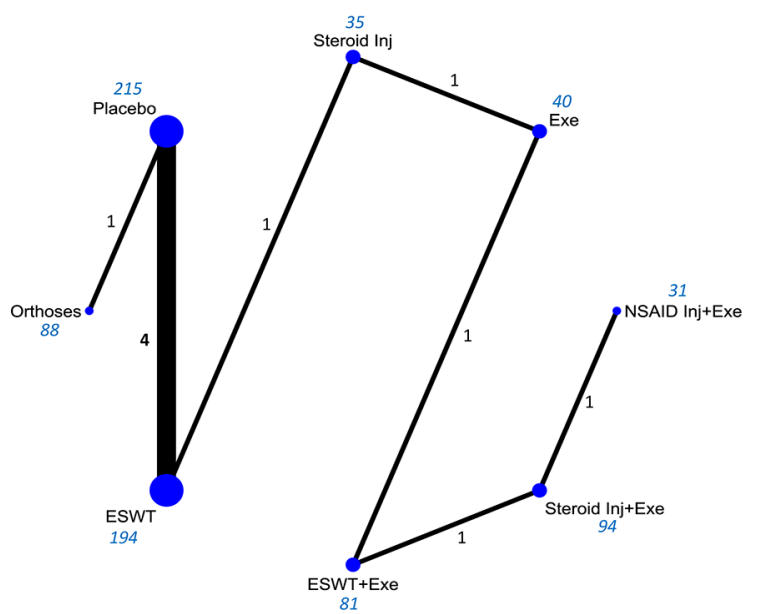

B

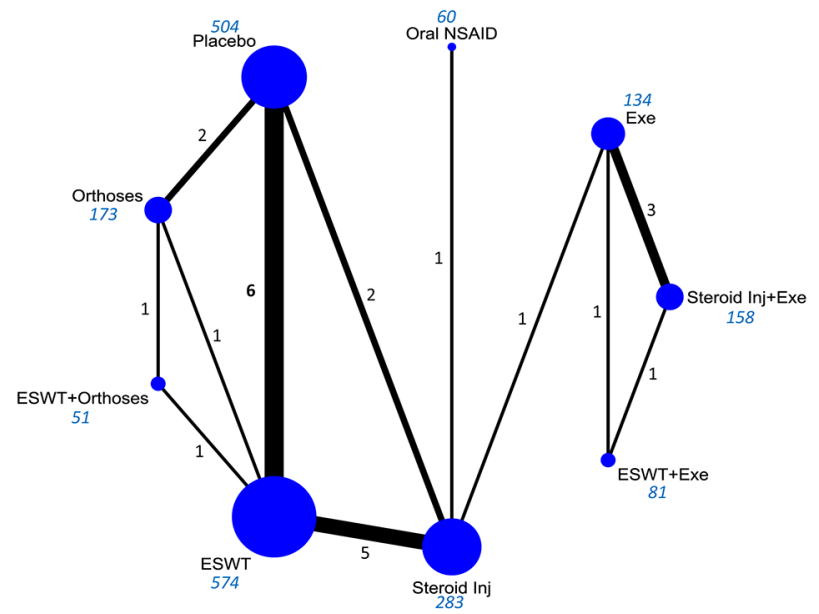

Figure 3 Network graph of included studies for pain outcomes, with thickness of lines and size of circles proportional to number of studies and number of participants, respectively. (A) Short-term evidence, (B) medium-term evidence and (C) long-term evidence. Black text represents number of studies, and blue text number of participants. ESWT, extracorporeal shockwave therapy; ESWT+Exe, extracorporeal shockwave therapy combined with exercise; ESWT+Orthoses, extracorporeal shockwave therapy combined with orthoses; Exe, exercise; NSAID Inj+Exe, oral non-steroidal antiinflammatory drug combined with exercise; Oral NSAID, oral non-steroidal anti-inflammatory drug; Orthoses, prefabricated or customised foot orthoses; Placebo, usual care/placebo; Steroid Inj, corticosteroid injection; Steroid Inj+Exe, corticosteroid injection combined with exercise.

data/reporting problems where authors could not be contacted or failed to respond to queries after repeated attempts over a 3 -month period $(n=17)$. Summary of findings and the characteristics of eligible studies that could not be incorporated into the meta-analysis are presented in online supplementary appendix 2,tables 1 and 2 .

Thirty-one RCTs involving 2450 participants across 10 different (combinations of) interventions (ESWT, ESWT+exercise, ESWT+orthoses, exercise, NSAID injection+exercise, oral NSAIDs, orthoses, corticosteroid injection, corticosteroid injection+exercise and placebo/sham) provided sufficient data for inclusion in the NMA. (online supplementary appendix 1 , table 1) presents the characteristics of the included RCTs. RCTs were published between 1999 and 2017. The maximum length of follow-up ranged from 4 weeks to 104 weeks. Most RCTs were from Europe $(n=8)$, followed by Asia $(n=6)$ and Australia $(n=5)$. RCTs recruited participants mostly from primary care sources and outpatient departments of hospitals and rehabilitation centres and investigated a combination of participants with duration of PHP symptoms ranging from 10 to 287 weeks.

\section{Risk of bias in the evidence base}

The risk of bias assessment for the 31 included trials is presented in figure 2A and B. All included studies were RCTs; however, a significant proportion (35\%) did not adequately report how randomisation was performed. High risk of bias was considered present most frequently (in 26\% of trials) in relation to lack of blinding of participants and personnel. Many of the trial outcomes were patient reported but outcome assessment procedures were reported as blinded in $45 \%$ of the trials. The reporting of most of the trials did not provide sufficient information to accurately assess concealment of treatment allocation, thus generating a large proportion of 'unclear' responses $(61 \%$ of trials). Overall, $14^{22} 26-38$ of the 31 trials were considered to 
A

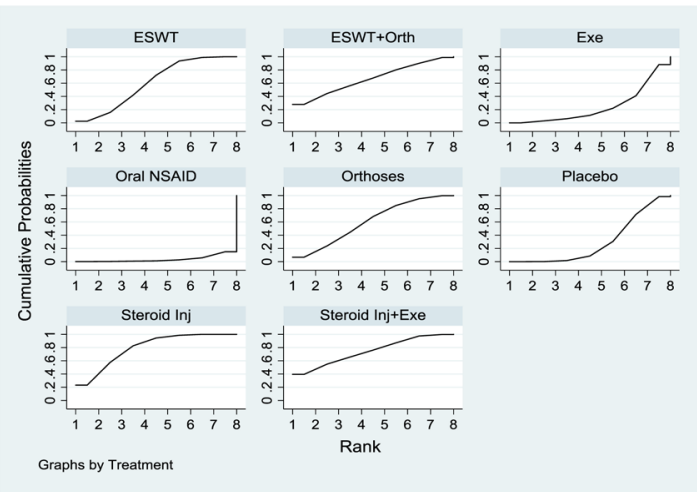

C

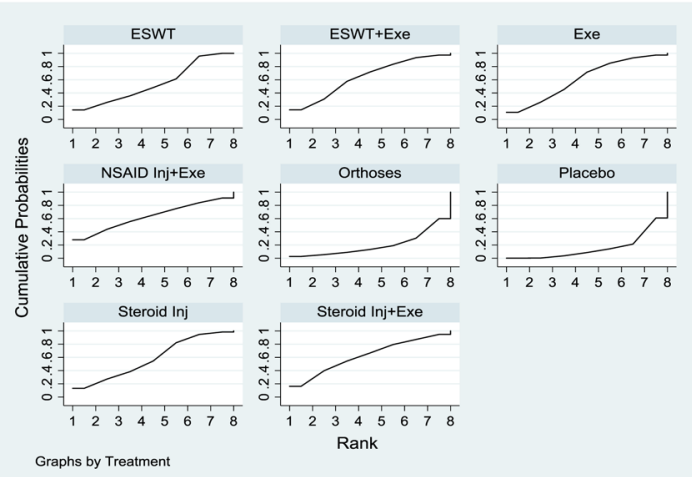

B

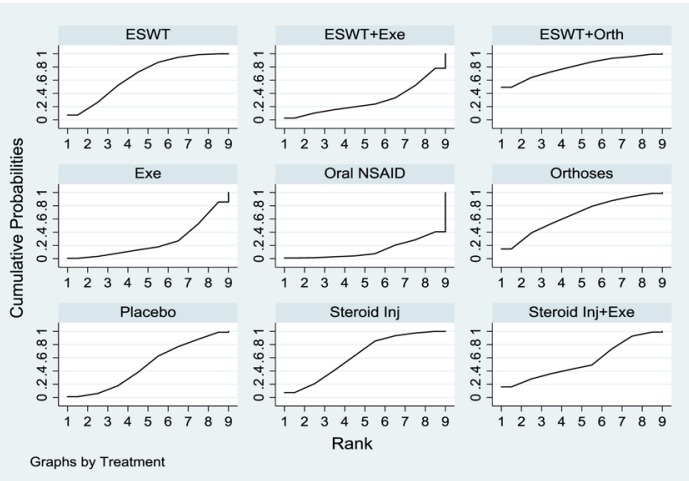

Figure 4 Cumulative ranking plots to show comparative effectiveness of treatments from a pain outcome network meta-analysis, for each of (A) short-term outcomes, (B) medium-term outcomes and (C) long-term outcomes. Results based on a simulation of 1000 replications. ESWT, extracorporeal shockwave therapy; ESWT+Exe, extracorporeal shockwave therapy combined with exercise; ESWT+Orthoses, extracorporeal shockwave therapy combined with orthoses; Exe, exercise; NSAID Inj+Exe, oral non-steroidal anti-inflammatory drug combined with exercise; Oral NSAID, oral non-steroidal anti-inflammatory drug; Orthoses, prefabricated or customised foot orthoses; Placebo, usual care/placebo; Steroid Inj, corticosteroid injection; Steroid Inj+Exe, corticosteroid injection combined with exercise.

be of low quality with fewer than $50 \%$ of risk of bias items (ie, $\leq 3 / 7$ ) classed as low risk.

\section{Network coherence (consistency and heterogeneity)}

NMA was possible for all (six) connected networks of evidence, which investigated pain and function outcomes separately, with follow-ups at: (i) short term, (ii) medium term and (iii) long term. There were no signs of the consistency assumption being violated for any network (where applicable, ie, only considering closed-loop networks). Firstly, the global Wald tests for inconsistency were not significant $(\mathrm{p}=0.822,0.971$, and 0.925 for short term pain, medium term pain, and short term function, respectively). Second, no statistically significant difference was observed between direct and indirect estimates when assessed separately for each treatment comparison through a node-splitting technique (all $\mathrm{p}$ values were $>0.05$ ). Third, the 95\% CIs of the network and pairwise meta-analysis summary results overlapped for all three closed-loop networks (online supplementary figure 1S). The heterogeneity term, $\tau^{2}$, was 'moderate' to 'large' in magnitude (as classed by Cohen's rule of thumb ${ }^{25}$ ) for all of the networks except long-term function (online supplementary appendix 1, table 2). Hence, random effects analyses were used for all but the long-term function network (whereby fixed effects analyses were used). Full raw outcome data used (including outcome scales) are provided in supplementary appendix 1, table 3 .

\section{Treatments for PHP: pain outcomes}

Evidence base: there were 22 studies $^{22} 26-2830313337$ 39-53 (21x two-arm, $1 \times 3 \mathrm{arm}$ ) in the short-term pain evidence base, with a

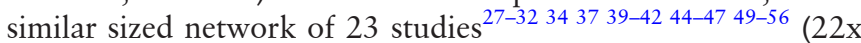
two-arm, $1 \times 3 \mathrm{arm})$ in the medium term pain, and a smaller network of 10 studies $^{29} 34-384044454955$ (all two-arm) in the long-term pain; as presented in figure 3. Eight different treatment nodes were used in the short-term analysis, with these same treatments and the addition of a ninth (ESWT+exercise) used in the medium term, and eight treatments in the long term. Placebo/sham-ESWT comparisons were most prevalent across all pain outcome networks ( $\mathrm{n}=6$ studies in short term and medium term, $n=4$ in long term), and the number of participants ranged from 31 (NSAID injection + exercise in long term) to 574 (ESWT in medium term). Direct evidence was available for 12 out of a possible 28 pairwise comparisons in the short term, 12/36 in the medium term and $7 / 28$ in the long term. Outcome follow-up ranged from 2 to 6 weeks in the short term $(n=1744$ total participants used), 2-3 months in the medium term $(\mathrm{n}=2018)$ and was fixed at 12 months for the long term $(n=778)$.

\section{Comparative effectiveness of treatments}

Full pairwise and network analyses results for pain are presented in table 1. Across both pairwise and network analyses, corticosteroid injection demonstrated a statistically significant larger reduction in short-term pain over oral NSAIDs (SMD 2.60, 


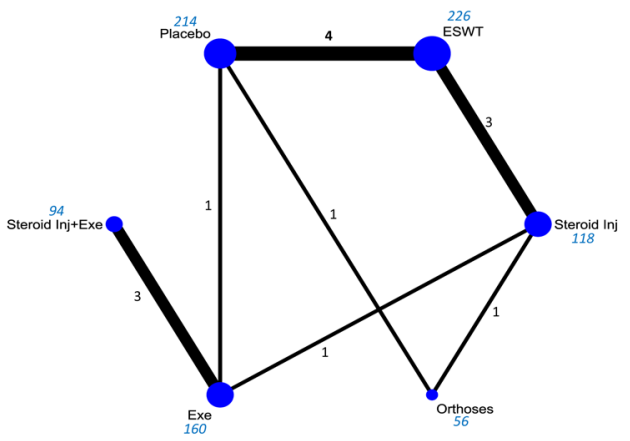

C

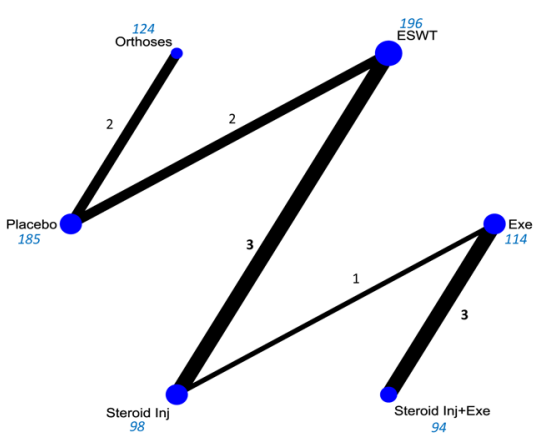

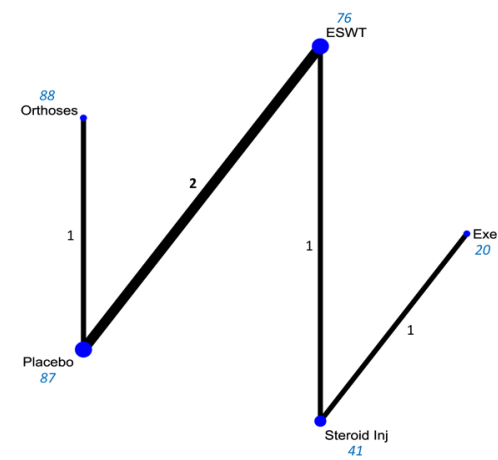

Figure 5 Network graph of included studies for function outcomes, with thickness of lines and size of circles proportional to number of studies and number of participants, respectively. (A) Short-term evidence, (B) medium-term evidence and C) long-term evidence. Black text represents number of studies, and blue text number of participants. ESWT, extracorporeal shockwave therapy; Exe, exercise; Orthoses, prefabricated or customised foot orthoses; Placebo, usual care/placebo; Steroid Inj, corticosteroid injection; Steroid Inj+Exe, corticosteroid injection combined with exercise.

95\% CI (0.81 to 4.39)); and corticosteroid injection combined with exercise showed a statistically significant larger reduction in pain compared with exercise alone (SMD 1.20, 95\% CI (0.14 to 2.26)). Compared with other treatments, oral NSAIDs were most often associated with the least statistically significantly reductions in short-term pain (by SMD 2.25, 95\% CI $(0.18$ to 4.33) compared with orthoses, and by SMD $2.61,95 \%$ CI $(0.13$ to 5.09) compared with corticosteroid injection combined with exercise).

Most treatments were not statistically significantly superior to one another and underlying estimates of effect presented with very wide CIs. For instance, the network comparison of ESWT combined with orthoses showed a non-statistically significant reduction in medium-term pain compared with ESWT in combination with exercise $(\mathrm{SMD}=2.36,95 \% \mathrm{CI}(-2.17$ to 6.89$))$.

With the highest SUCRA values of 79.5 and 74.4 , and the best mean ranks of 2.4 and 2.8, corticosteroid injection alone and in combination with exercise ranked among the three most effective treatments for short-term pain, $82.7 \%$ and $65.7 \%$ of the time, respectively (figure $4 \mathrm{~A}$, table 2 ). In contrast, oral NSAIDs (which ranked among the three least effective treatments $97.3 \%$ of the time), exercise alone, and placebo/sham interventions demonstrated the least comparative effectiveness for pain relief in the short term. General trends from the NMA and direct comparisons for medium-term pain indicated that ESWT combined with orthoses may be more effective than other treatments (highest SUCRA value of 80.3 ; figure $4 \mathrm{~B}$, table 2). Oral NSAIDs, exercise and exercise combined with ESWT were least likely to have beneficial effects for the treatment of pain due to PHP in the medium term compared with other treatments. While placebo and orthoses appeared least likely to be beneficial for long-term pain $(85.7 \%$ and $81.0 \%$ of the time ranking among three least effective treatments, respectively; figure $4 \mathrm{C}$, table 2 ), superiority of one treatment over another for the remaining six treatments was less clear, with most of these treatments having similar rankings (average SUCRA of 60.8).

\section{Treatments for PHP: function outcomes}

Evidence base: for function outcomes, there were fewer RCTs available for analysis compared with the pain (14 studies were in the network for short-term function, ${ }^{26} 3033373941-4648495253$ 11 for medium-term function, $3037414244-4649525355$ and 5 for long-term function 3537444955 ; all two-armed), as shown in figure 5. Similar treatment nodes were used across the networks, with the same six used in short-term and medium-term function analyses (ESWT, ESWT+exercise, orthoses, placebo, corticosteroid injection with and without exercise), while the long-term analysis did not contain corticosteroid without exercise. Placebo/ sham-ESWT comparisons were most common in the short term ( $\mathrm{n}=4$ studies) and long term ( $\mathrm{n}=2$ studies), while ESWT/corticosteroid injection and corticosteroid injection with exercise/exercise alone comparisons ( $\mathrm{n}=3$ studies) were joint most common for medium term. The number of participants ranged from 20 

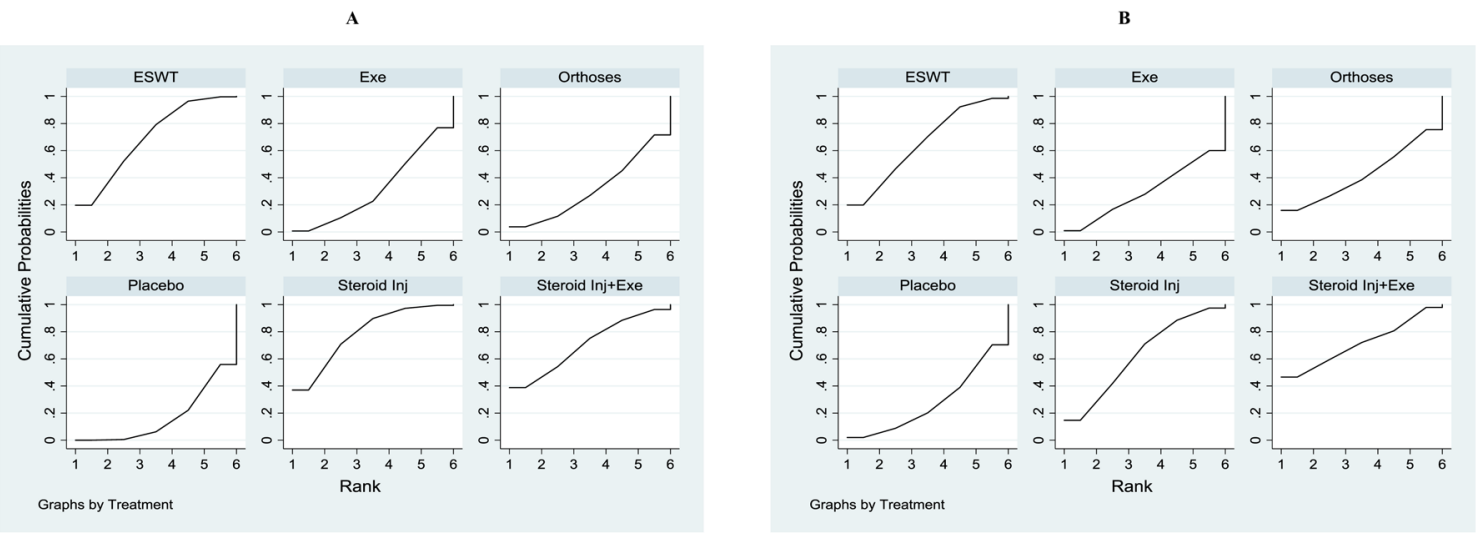

C

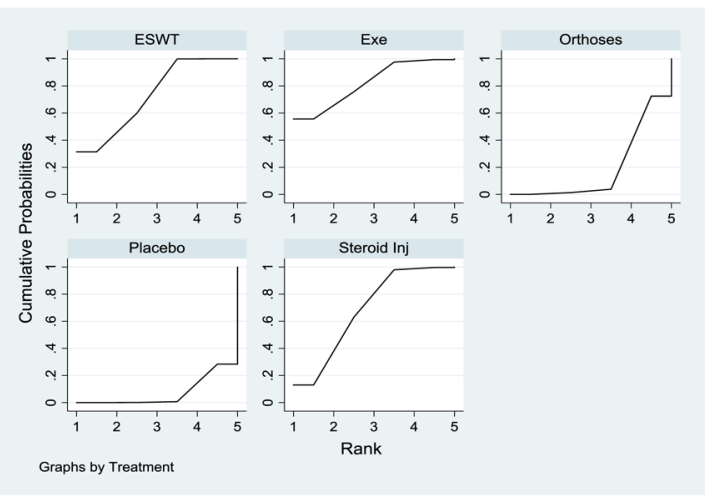

Figure 6 Cumulative ranking plots to show comparative effectiveness of treatments from a function outcome network meta-analysis, for each of (A) short-term outcomes, (B) medium-term outcomes and (C) long-term outcomes. Results based on a simulation of 1000 replications. ESWT, extracorporeal shockwave therapy; Exe, exercise; Orthoses, prefabricated or customised foot orthoses; Placebo, usual care/placebo; Steroid Inj, corticosteroid injection; Steroid Inj+Exe, corticosteroid injection combined with exercise.

(exercise in long term) to 226 (ESWT in short term), and direct evidence was available for 7 out of a possible 15, 5/15 and 4/10 comparisons, in the short term, medium term and long term, respectively. Outcome follow-up ranged from 2 to 6 weeks in the short term ( $\mathrm{n}=868$ total participants used), 2.5-3 months in the medium term $(n=811)$ and was fixed at 12 months for the long term $(n=312)$.

\section{Comparative effectiveness of treatments}

The comparative effectiveness of treatments (both pairwise and network meta-analyses) on function outcomes are presented in table 3 . As with pain outcomes, most treatments were not significantly better than one another in the short term, medium term and long term; CIs were often wide. Placebo/sham interventions were comparatively worse at improving functional ability than other treatments, for example, NMA showed statistically significant reductions in long-term functional ability (by SMD 0.93, $95 \% \mathrm{CI}(0.23$ to 1.63$)$ compared with corticosteroid injection, by SMD $1.09,95 \%$ CI $(0.15$ to 2.03$)$ compared with exercise and by SMD $0.95,95 \%$ CI (0.50 to 1.40$)$ compared with ESWT).

In agreement with the analyses on pain outcome treatment effects, placebo/sham interventions ranked least likely to improve function for patients with PHP (SUCRA values: 16.9, 28.1 and 7.3 , in the short term, medium term and long term, respectively; figure 6 and table 4), followed by orthoses (SUCRA: 31.8 , 42.4 and 19.4 , in the short term, medium term and long term,

Table 4 Network meta-analysis treatment ranking results for function outcome analyses, for each of short-term outcomes, medium-term outcomes and long-term outcomes

\begin{tabular}{|c|c|c|c|c|c|c|}
\hline \multirow[b]{2}{*}{ Treatment } & \multicolumn{2}{|c|}{ Short-term function } & \multicolumn{2}{|c|}{ Medium-term function } & \multicolumn{2}{|c|}{ Long-term function } \\
\hline & SUCRA & Mean rank & SUCRA & Mean rank & SUCRA & Mean rank \\
\hline ESWT & 69.5 & 2.5 & 65.6 & 2.7 & 72.8 & 2.1 \\
\hline Exe & 32.2 & 4.4 & 29.9 & 4.5 & 82.1 & 1.7 \\
\hline Orthoses & 31.8 & 4.4 & 42.4 & 3.9 & 19.4 & 4.2 \\
\hline Placebo & 16.9 & 5.2 & 28.1 & 4.6 & 7.3 & 4.7 \\
\hline Steroid Inj & 78.9 & 2.1 & 62.7 & 2.9 & 68.4 & 2.3 \\
\hline Steroid Inj+Exe & 70.6 & 2.5 & 71.4 & 2.4 & & \\
\hline
\end{tabular}

Surface under cumulative ranking curve (SUCRA) values $(0-100)$ and mean ranks are presented, based on a simulation with 1000 replications. Higher SUCRAs and lower mean ranks indicate better performing treatments.

ESWT, extracorporeal shockwave therapy; Exe, exercise; Orthoses, prefabricated or customised foot orthoses; Placebo, usual care/placebo; Steroid Inj, corticosteroid injection; Steroid Inj+Exe, corticosteroid injection combined with exercise. 

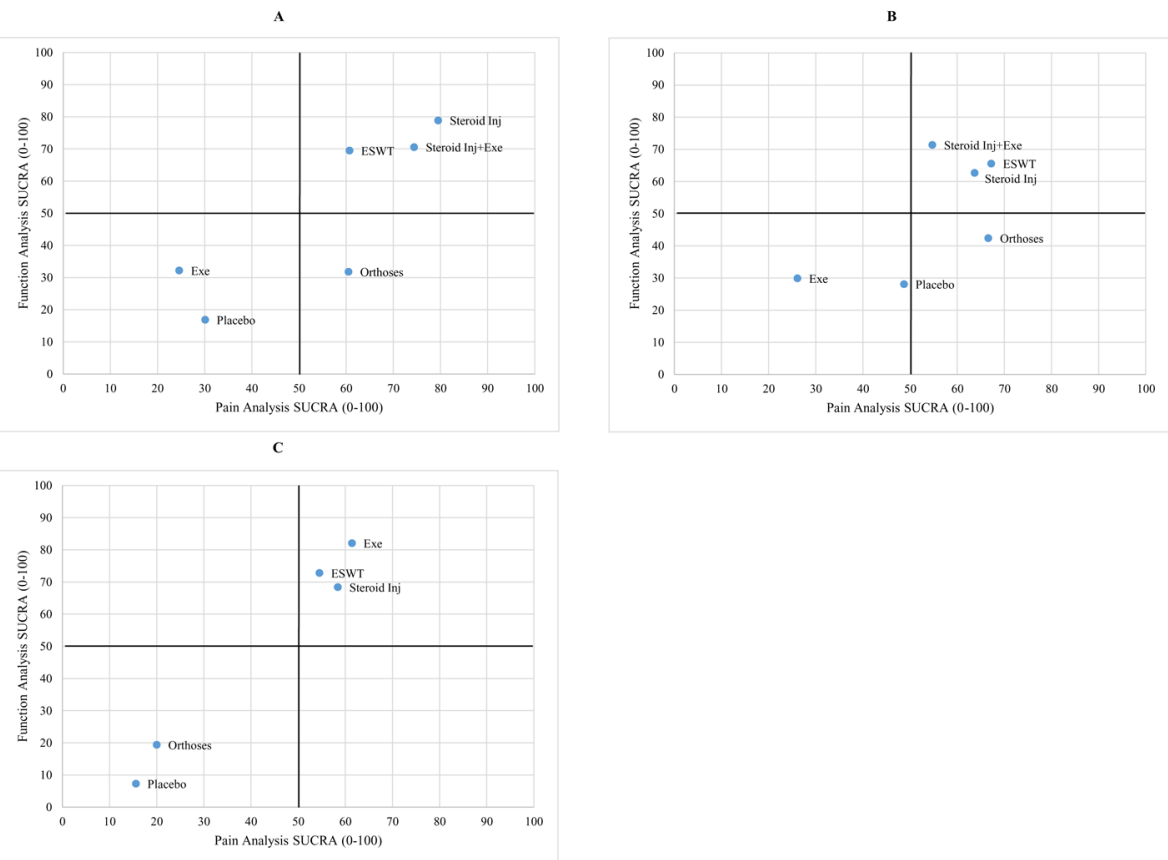

Figure 7 Scatter plots to show comparative effectiveness of treatments ${ }^{*}$, through surface under cumulative ranking curve (SUCRA) values (0-100), for pain (X-axis) and function (Y-axis) outcomes. Shown separately for each of: A) short-term outcomes, (B) medium-term outcomes and C) longterm outcomes. Higher SUCRAs indicate better performing treatments. Horizontal and vertical lines added at SUCRA=50 values as a crude guide to identifying comparatively better/worse performing treatments for pain/function. ESWT, extracorporeal shockwave therapy; Exe, exercise; Orthoses, prefabricated or customised foot orthoses; Placebo, usual care/placebo; Steroid Inj, corticosteroid injection; Steroid Inj+Exe, corticosteroid injection combined with exercise. *SUCRA results for four treatments are completely omitted, as data were only available for pain, but not function outcomes. ESWT+Exe, extracorporeal shockwave therapy combined with exercise; ESWT+Orthoses, extracorporeal shockwave therapy combined with orthoses; NSAID Inj+Exe, oral non-steroidal anti-inflammatory drug combined with exercise; Oral NSAID, oral non-steroidal anti-inflammatory drug.

respectively), and exercise alone (SUCRA: 32.2 and 29.9 in the short term and medium term, respectively). However, exercise appeared most likely to improve functional ability for long-term function (SUCRA: 82.1); while corticosteroid with and without exercise, and ESWT consistently ranked in the top three treatments most likely to improve functional ability.

\section{Comparison of effectiveness of treatments across pain and function outcomes}

Corticosteroid injection with and without exercise, and ESWT interventions appear most likely to have beneficial effects for both pain and function outcomes over all time periods (figure 7). In contrast, placebo/sham interventions appear least likely to improve either pain or function outcomes across all time periods, while exercise appears to have a non-beneficial effect for short term and medium term, but a beneficial effect for long-term pain and function.

\section{Summary of findings for RCTs without suitable data for NMA}

Findings from 17 RCTs of 7 different comparisons and/or treatment combinations, including ESWT versus placebo/sham $(n=11)$, exercise versus ESWT $(n=1)$ and custom versus prefabricated orthosis/placebo/sham $(n=3)$, for which suitable data could not be obtained are presented in online supplementary appendix 2, table 1. For the comparison between ESWT and placebo/sham, with an unclear to high risk of bias across trials, ESWT is reported to be significantly more effective than sham/ placebo for reducing pain in two out of three trials in the short term, and four out of seven in the medium term. There was no evidence for the effect of ESWT on function in the short term but two trials reported reduction in functional disability in the medium term. However, there was uncertainty in evidence across trials and time points as shown by very large CIs and inconsistency of the magnitude of effects. For both pain and function outcomes and across time points (short term, medium term and long term), trials found no difference between custom and prefabricated orthoses. All other treatment comparisons/combinations contained only one trial with mostly small sample sizes.

\section{DISCUSSION}

Available evidence does not suggest that any of the commonly used treatments for the management of PHP are significantly better than any other, although the results of this NMA show that corticosteroid injections alone or in combination with exercise are effective treatments for reducing pain and improving function in the short term. However, the magnitude of estimate of effect varied widely across trials with large CIs. Furthermore, the overall effect of corticosteroid injections on PHP is modest, and the potential for adverse effects ${ }^{15} 57$ such as postinjection steroid-induced increase in pain, fat pad atrophy, nerve injury and rupture of the plantar fascia require careful consideration. There was a greater amount of evidence for ESWT, but we found no evidence that this treatment confers more beneficial effects (compared with the other treatments in this study) for reducing pain and improving function among patients with PHP.

In the network meta-analyses of both pain and function, placebo/sham interventions and NSAIDs were generally shown to be the least effective treatment options. Considering PHP has long been considered to be a self-limiting pain condition, our findings indicate that first-line management recommendations of PHP with over the counter pain medications, NSAIDs and a watchful waiting approach may be suboptimal. Previous literature has suggested that 
delaying treatment may worsen prognosis, and potentially create a need for further healthcare use. ${ }^{57}$ The findings of this present study support the notion that access to treatments without a period of watchful waiting may be beneficial.

As the current NMA is the first to examine the comparative effectiveness of the most common treatments for PHP, it is difficult to directly compare the findings of the present study with those of previous NMAs which examined a limited number of treatments, ${ }^{58}$ or compared dosage/technique for specific treatment options. ${ }^{59}$ Previous reviews collectively indicate that exercise and foot orthoses are promising interventions for short-term and medium-term improvements in pain and function. ${ }^{6061} \mathrm{In}$ this review, exercise as a stand-alone treatment was not found to consistently confer beneficial effects in reducing pain and improving function for patients with PHP in the short term, but a beneficial effect was found for long-term pain and function. There is a lack of evidence regarding the most effective exercise dose or delivery method. In this systematic review, included RCTs reported varying exercise therapy protocols, dose and regime. As with the review by Almubarak and Foster, ${ }^{60}$ exercise as a treatment in this review included stretching and strengthening exercise trials; treatment comparisons including exercise in combination with other treatments such as corticosteroid injection mostly had calf stretching as the 'exercise' component. These exercises were mostly home based (apart from the first session that may be supervised) and were not individualised or progressed. Within the networks, foot orthoses (prefabricated or custom), were not found to be effective as a stand-alone treatment for PHP, but were mostly effective in combination with ESWT. Our findings agree with those of recent systematic reviews showing that foot orthoses are better than sham/placebo and may be effective for reducing pain in the medium term. ${ }^{13} 61$

\section{Study strengths and limitations}

In this study, direct and indirect evidence has been combined in order to assess comparative effectiveness of interventions that have not yet (or only minimally) been directly compared in robust high-quality trials. There was agreement between the direct and indirect evidence which achieved consistency for specified treatments; however, tests for inconsistency are likely to be underpowered, due to lack of data, as evidenced by wide 95\% CIs for SMDs. As an alternative to frequentist methods which was used in the current NMA, a Bayesian three-level hierarchical NMA model may be employed. This approach has been shown to increase precision of effect estimates in meta-analysis of few trials, or a large number of treatment options which can be further subdivided. ${ }^{62}$ However, this approach was deemed to be out of scope for our NMA which mainly focusses on comparisons across different treatments. Future NMAs, especially those incorporating dose comparisons and procedural variations of the same treatment options, would benefit from Bayesian analysis.

The current study is not without limitations and must be interpreted with caution. First is the inclusion of only the most common treatments as opposed to all available treatments for the management of PHP. This decision was made in order to inform choice of treatment in primary care settings where PHP patients are mostly seen, and to evaluate interventions that are widely available and accessible to patients. Furthermore, networks would likely be disconnected when including a large number of treatments evaluated in only a small number of trials. The sparsity of data did not allow for a statistical exploration of publication bias; however, we conducted a comprehensive search of published and unpublished literature as well as employed a paired screening process to ensure all available evidence was identified. However, the findings of this review are still likely to be influenced by the small number of trials (mostly with small sample sizes) available to support direct and indirect comparisons in the network. For instance, many nodes in the networks (figure 3 and figure 5), were connected by only a single trial and (for some treatments) with few participants.

The loss of data associated with absence of suitable data for analysis was a challenge in this review. Related first, and more importantly, to the disparate reporting of data in scientific reports in this field, a lot of data from otherwise eligible (but excluded trials) could not be analysed mostly due to lack of reporting of treatment outcomes with a mean as well as a measure of variability. Despite concerted efforts to request this additional data from trial authors, the inability of our review to incorporate such data into evidence synthesises inadvertently led to notable research waste. As a minimum, for all trials in this field, reporting an average and a measure of variability (eg, a mean and a SD) per trial arm for each follow-up period should be required. Furthermore, to avoid substantial heterogeneity, data from some trials which used a very different approach to measuring outcomes could not be combined in the network. However, this problem could be overcome through the development of and adherence to an agreed standardised set of core outcomes to be used in trials in this field. In order to minimise the loss of potentially useful evidence, details of all otherwise eligible trials were extracted with a narrative summary of findings presented (online supplementary appendix 2, table 1). Generally, the results from these trials were found to be in agreement with the evidence presented in the NMA.

\section{Implications for clinical practice, policy and future research}

Within the NMA, control treatments (including placebo/sham interventions, watchful waiting approach, over-the-counter pain medications) and NSAIDs generally showed lack of beneficial effects for patients with PHP. For primary care first-point-ofcontact decision-making purposes, our findings suggest that access to treatments may be beneficial for patients with PHP.

However, findings from this review must be interpreted with caution due to limitations in quality of the evidence underpinning the analyses. Of particular concern are predominantly small sample sizes, low quality reporting of aspects of study design (especially concealment of treatment allocation) and variability in outcome measures across included studies (online supplementary appendix 1, table 3). Furthermore, this review cannot comment on evidence for comparative effectiveness of treatment options where the influence of duration of symptoms prior to treatment may be of concern. This is due to the wide variability in the range of duration of symptoms at recruitment across studies included in this review and the fact that most trials did not report data regarding the duration of symptoms per trial arm. Future research involving patients with PHP should therefore focus on the design of large trials with head-to-head comparisons of active treatments, long-term follow-up and higher reporting standards. Furthermore, careful consideration of trials investigating the same treatment comparisons (especially for the most promising interventions in the short term and long term) is an important next step. This will enable exploration of the optimal mode of delivery, dosage and intensity of treatments required for successful management of PHP.

\section{CONCLUSION}

This is the first NMA to examine the comparative effectiveness of commonly used treatments for PHP and brings together available evidence in order to aid evidence-informed clinical decisions in 
What is already known on this topic?

- Existing pairwise meta-analyses are limited to comparisons of two or three treatment options for plantar heel pain.

- Clinical decision making regarding the best treatment option is often difficult.

the management of PHP. For pain and functional outcomes, most treatments were not significantly better than others in the short term, medium term and long term. The comparative effectiveness of commonly used treatments (ie, exercise therapy, corticosteroid injections, orthoses, NSAIDs and ESWT) is limited by large variation in magnitude and imprecision of effect estimates. Findings indicate the need for large, multicentre trials directly comparing commonly used treatments for the management of PHP.

Acknowledgements The authors would like to thank Dr Nadia Corp for her help with the search strategy.

Contributors Investigation: OOB, MT, CL, LSC, AL, HBM, ER, DvdW. Formal analysis: OOB, AL. Writing —original draft: OOB, AL. Writing —review and editing: OOB, AL, CL, LSC, MT, HBM, ER, DvdW. Supervision: DvdW, ER. Project administration: OOB, DvdW.

Funding AL is supported by a National Institute for Health Research (NIHR) Research Methods Fellowship. MJT has received funding through a NIHR School for Primary Care Research Launching Fellowship and is currently supported by a Health Education England (HEE)/NIHR Integrated Clinical Academic Programme Clinical Lectureship (ICA-CL-2016-02-014).

Disclaimer The views expressed in this publication are those of the author(s) and not necessarily those of the NHS, the NIHR, HEE or the Department of Health and Social Care

Competing interests None daclared.

Patient consent Not required.

Provenance and peer review Not commissioned; externally peer reviewed.

\section{REFERENCES}

1 Landorf KB. Plantar heel pain and plantar fasciitis. BMJ Clin Evid 2015;2015:1111.

2 Taunton JE, Ryan MB, Clement DB, et al. A retrospective case-control analysis of 2002 running injuries. Br I Sports Med 2002;36:95-101.

3 Riddle DL, Pulisic M, Pidcoe P, et al. Risk factors for Plantar fasciitis: a matched casecontrol study. J Bone Joint Surg Am 2003;85-A:872-7.

4 Rome K, Howe T, Haslock I. Risk factors associated with the development of plantar heel pain in athletes. Foot 2001;11:119-25.

\section{What are the new findings?}

- For the management of plantar heel pain, available evidence does not support the superiority of any of the commonly available treatments over another.

- However, corticosteroid injections, alone or in combination with exercise, and ESWT appear more likely to be effective for relieving plantar heel pain and improving function compared with other treatments in the short term, medium term and long term.

- Control treatments (which include over-the-counter pain medications and watchful waiting, as well as placebo interventions) generally show less beneficial effects than other treatments for patients with plantar heel pain.

- The review highlights the need for large high-quality randomised controlled trials of the commonly used interventions for the management of plantar heel pain.
5 Irving DB, Cook JL, Menz HB. Factors associated with chronic plantar heel pain: a systematic review. I Sci Med Sport 2006;9:11-22.

6 Orchard J. Plantar fasciitis. BMJ 2012;345:e6603.

7 Tong KB, Furia J. Economic burden of plantar fasciitis treatment in the United States. Am J Orthop 2010;39:227-31.

8 Map of Medicine. Plantar Fasciitis. $2014 \mathrm{http}: / /$ healthquides.mapofmedicine.com/ choices/map/plantar_fasciitis1.html (accessed 07 Nov 2017).

9 Clinical Knowledge Summaries (CKS). Plantar Fasciitis: National Institute for Health and Care Excellence. 2014 http://cks.nice.org.uk/plantar-fasciitis (accessed 07 Nov 2017).

10 Lee SY, McKeon P, Hertel J. Does the use of orthoses improve self-reported pain and function measures in patients with plantar fasciitis? A meta-analysis. Phys Ther Sport 2009;10:12-18.

11 Young CC, Rutherford DS, Niedfeldt MW. Treatment of plantar fasciitis. Am Fam Physician 2001;63:46777-748.

12 Crawford F, Thomson C. Interventions for treating plantar heel pain. Cochrane Database Syst Rev 2003:CD000416.

13 Salvioli S, Guidi M, Marcotulli G. The effectiveness of conservative, nonpharmacological treatment, of plantar heel pain: A systematic review with metaanalysis. Foot 2017;33:57-67.

14 Hawke F, Burns J, Radford JA, et al. Custom-made foot orthoses for the treatment of foot pain. Cochrane Database Syst Rev 2008;3:CD006801 http://onlinelibrary.wiley. com/doi/.

15 David JA, Sankarapandian V, Christopher PR, et al. Injected corticosteroids for treating plantar heel pain in adults. Cochrane Database Syst Rev 2017;6:CD009348 http:// onlinelibrary.wiley.com/doi/.

16 Sweeting D, Parish B, Hooper L, et al. The effectiveness of manual stretching in the treatment of plantar heel pain: a systematic review. J Foot Ankle Res 2011;4:19.

17 Salanti G. Indirect and mixed-treatment comparison, network, or multiple-treatments meta-analysis: many names, many benefits, many concerns for the next generation evidence synthesis tool. Res Synth Methods 2012;3:80-97.

18 Hutton B, Salanti G, Caldwell DM, et al. The PRISMA extension statement for reporting of systematic reviews incorporating network meta-analyses of health care interventions: checklist and explanations. Ann Intern Med 2015;162:777-84.

19 Grieve R, Palmer S. Physiotherapy for plantar fasciitis: a UK-wide survey of current practice. Physiotherapy 2017;103:193-200 https://doi.org/.

20 Higgins JP, Altman DG, Gøtzsche PC, et al. The Cochrane Collaboration's tool for assessing risk of bias in randomised trials. BMJ 2011;343:d5928.

21 Wan X, Wang W, Liu J, et al. Estimating the sample mean and standard deviation from the sample size, median, range and/or interquartile range. BMC Med Res Methodol 2014;14:135.

22 Walther M, Kratschmer B, Verschl J, et al. Effect of different orthotic concepts as first line treatment of plantar fasciitis. Foot Ankle Surg 2013;19:103-7.

23 White IR, Barrett JK, Jackson D, et al. Consistency and inconsistency in network metaanalysis: model estimation using multivariate meta-regression. Res Synth Methods 2012;3:111-25.

24 Dias S, Welton NJ, Caldwell DM, et al. Checking consistency in mixed treatment comparison meta-analysis. Stat Med 2010 8:932-44.

25 Cohen J. Statistical power analysis for the behavioral sciences. Hilsdale. NJ: Lawrence Earlbaum Associates, 1988.

26 Hawamdeh Z, Alghwiri AA, Nassar A. The short-term effect of extracorporeal shock wave in treating plantar fasciitis: RCT. Jordan Medical Journal 2016;50:1-11.

27 Mardani-Kivi M, Karimi Mobarakeh M, Hassanzadeh Z, et al. Treatment Outcomes of Corticosteroid Injection and Extracorporeal Shock Wave Therapy as Two Primary Therapeutic Methods for Acute Plantar Fasciitis: A Prospective Randomized Clinical Trial. J Foot Ankle Surg 2015;54:1047-52.

28 Yan W, Sun S, Li X. [Therapeutic effect of extracorporeal shock wave combined with orthopaedic insole on plantar fasciitis]. Zhong Nan Da Xue Xue Bao Yi Xue Ban 2014;39:1326-30.

29 Grecco MV, Brech GC, Greve JM. One-year treatment follow-up of plantar fasciitis: radial shockwaves vs. conventional physiotherapy. Clinics 2013;68:1089-95.

30 Ryan M, Hartwell J, Fraser S, et al. Comparison of a physiotherapy program versus dexamethasone injections for plantar fasciopathy in prolonged standing workers: a randomized clinical trial. Clin I Sport Med 2014;24:211-7.

31 Biswas C, Pal A, Acharya A. A comparative study of efficacy of oral nonsteroidal antiinflammatory agents and locally injectable steroid for the treatment of plantar fasciitis. Anesth Essays Res 2011;5:158-61.

32 Yucel I, Ozturan KE, Demiraran Y, et al. Comparison of high-dose extracorporeal shockwave therapy and intralesional corticosteroid injection in the treatment of plantar fasciitis. J Am Podiatr Med Assoc 2010;100:105-10.

33 Chow IH, Cheing GL. Comparison of different energy densities of extracorporeal shock wave therapy (ESWT) for the management of chronic heel pain. Clin Rehabil 2007;21:131-41.

34 Porter MD, Shadbolt B. Intralesional corticosteroid injection versus extracorporeal shock wave therapy for plantar fasciopathy. Clin I Sport Med 2005;15:119-24.

35 Rompe JD, Decking J, Schoellner C, et al. Shock wave application for chronic plantar fasciitis in running athletes. A prospective, randomized, placebo-controlled trial. Am J Sports Med 2003;31:268-75. 
36 Lizis P. Comparison between Real and Placebo Extracorporeal Shockwave Therapy for the Treatment of Chronic Plantar Fasciitis Pain in the Males. Iran J Public Health 2015;44:1150.

37 Guevara Serna JA, Acosta Moron JA. Revista Colombiana de Ortopedia y Traumatologia, 2017.

38 Guner S, Onder H, Guner SI, et al. Effectiveness of local tenoxicam versus corticosteroid injection for plantar fasciitis treatment. Orthopedics 2013;36:e132 2-e1326.

39 Buchbinder R, Ptasznik R, Gordon J, et al. Ultrasound-guided extracorporeal shock wave therapy for plantar fasciitis: a randomized controlled trial. JAMA 2002;288:1364-72

40 Haake M, Buch M, Schoellner C, et al. Extracorporeal shock wave therapy for plantar fasciitis: randomised controlled multicentre trial. BMJ 2003;327:75.

41 McMillan AM, Landorf KB, Gilheany MF, et al. Ultrasound guided corticosteroid injection for plantar fasciitis: randomised controlled trial. BMJ 2012;344:e3260.

42 Oliveira HA, Jones A, Moreira E, et al. Effectiveness of total contact insoles in patients with plantar fasciitis. J Rheumatol 2015;42:870-8.

43 Radford JA, Landorf KB, Buchbinder R, et al. Effectiveness of calf muscle stretching for the short-term treatment of plantar heel pain: a randomised trial. BMC Musculoskelet Disord 2007;8:36

44 Ibrahim MI, Donatelli RA, Schmitz C, et al. Chronic plantar fasciitis treated with two sessions of radial extracorporeal shock wave therapy. Foot Ankle Int 2010;31:391-7.

45 Ibrahim MI, Donatelli RA, Hellman M, et al. Long-term results of radial extracorporeal shock wave treatment for chronic plantar fasciopathy: A prospective, randomized, placebo-controlled trial with two years follow-up. J Orthop Res 2017;35:1532-8.

46 Mahindra P, Yamin M, Selhi HS, et al. Chronic Plantar Fasciitis: Effect of Platelet-Rich Plasma, Corticosteroid, and Placebo. Orthopedics 2016;39:e285-e289.

47 Theodore GH, Buch M, Amendola A, et al. Extracorporeal shock wave therapy for the treatment of plantar fasciitis. Foot Ankle Int 2004;25:290-7.

48 Yucel U, Kucuksen S, Cingoz HT, et al. Full-length silicone insoles versus ultrasoundguided corticosteroid injection in the management of plantar fasciitis: a randomized clinical trial. Prosthet Orthot Int 2013;37:471-6.

49 Celik D, Kuş G, Sırma SÖ. Joint Mobilization and Stretching Exercise vs Steroid Injection in the Treatment of Plantar Fasciitis: A Randomized Controlled Study. Foot Ankle Int 2016:37:150-6.
50 Crawford F, Atkins D, Young P, et al. Steroid injection for heel pain: evidence of shortterm effectiveness. A randomized controlled trial. Rheumatology 1999;38:974-7.

51 Ball EM, McKeeman HM, Patterson C, et al. Steroid injection for inferior heel pain: a randomised controlled trial. Ann Rheum Dis 2013;72:996-1002.

52 Hocaoglu S, Vurdem UE, Cebicci MA, et al. Comparative Effectiveness of Radial Extracorporeal Shockwave Therapy and Ultrasound-Guided Local Corticosteroid Injection Treatment for Plantar Fasciitis. J Am Podiatr Med Assoc 2017;107:192-9.

53 Eslamian F, Shakouri SK, Jahanjoo F, et al. Extra Corporeal Shock Wave Therapy Versus Local Corticosteroid Injection in the Treatment of Chronic Plantar Fasciitis, a Single Blinded Randomized Clinical Trial. Pain Med 2016;17:1722-31.

54 Kudo P, Dainty K, Clarfield M, et al. Randomized, placebo-controlled, double-blind clinical trial evaluating the treatment of plantar fasciitis with an extracoporeal shockwave therapy (ESWT) device: a North American confirmatory study. J Orthop Res 2006;24:115-23.

55 Landorf KB, Keenan AM, Herbert RD. Effectiveness of foot orthoses to treat plantar fasciitis: a randomized trial. Arch Intern Med 2006;166:1305-10.

56 Vahdatpour B, Sajadieh S, Bateni V, et al. Extracorporeal shock wave therapy in patients with plantar fasciitis. A randomized, placebo-controlled trial with ultrasonographic and subjective outcome assessments. J Res Med Sci 2012; 17:834-8.

57 Wolgin M, Cook C, Graham C, et al. Conservative treatment of plantar heel pain: long-term follow-up. Foot Ankle Int 1994;15:97-102.

58 Hsiao MY, Hung CY, Chang KV, et al. Comparative effectiveness of autologous bloodderived products, shock-wave therapy and corticosteroids for treatment of plantar fasciitis: a network meta-analysis. Rheumatology 2015;54:1735-43.

59 Tsikopoulos K, Vasiliadis HS, Mavridis D. Injection therapies for plantar fasciopathy ('plantar fasciitis'): a systematic review and network meta-analysis of 22 randomised controlled trials. Br J Sports Med 2016:50:1367-75.

60 Almubarak AA, Foster N. Exercise Therapy for Plantar Heel Pain: A Systematic Review. IJES 2012;5:9.

61 Whittaker GA, Munteanu SE, Menz HB, et al. Foot orthoses for plantar heel pain: a systematic review and meta-analysis. Br J Sports Med 2018;52:e40.

62 Owen RK, Tincello DG, Keith RA. Network meta-analysis: development of a three-level hierarchical modeling approach incorporating dose-related constraints. Value Health 2015:18:116-26. 